\title{
PERFORMANCE OF THE LEP2 SRF SYSTEM
}

\author{
D. Boussard, CERN, Switzerland
}

\section{Abstract}

In 1996, LEP2 operated at an energy of up to $86 \mathrm{GeV}$ per beam using, in addition to the conventional RF system, 176 newly installed superconducting (SC) cavities, which together provide up to $2 \mathrm{GV}$ per turn at $352 \mathrm{MHz}$. Almost all SC cavities are of the niobium film on copper type; they ran at an average operating gradient of $6 \mathrm{MV} / \mathrm{m}$ with a total beam current of up to $5.5 \mathrm{~mA}$. The behaviour of SC cavities and couplers has been very satisfactory: only two cavities out of 176 were field limited; they have however been recovered in situ since. Small modifications in the liquid helium distribution reduced to a large extent turbulent phenomena and the associated microphonic effects. However the intrinsic electroacoustic instabilities (ponderomotive oscillations) remain the major concern for the operation of the RF system at high intensity. Their effect on the beam is minimized using RF feedback (on the vector sum of individual cavity signals); this also suppresses the intensity limitations due to beam loading instabilities. Installation, commissioning and operation of a huge new RF power plant (24 MW installed RF power) went as expected, despite a few weak points which are now being fixed.

\section{INTRODUCTION}

LEP, the largest particle accelerator in the world, is an electron-positron collider which started operation in 1989 at a collision energy of $45 \mathrm{GeV}$ (the $\mathrm{Z}_{0}$ energy) with a room-temperature RF system operationally capable of delivering up to $340 \mathrm{MV}$ at $352 \mathrm{MHz}$.

The so-called LEP2 programme started in 1991 was aimed at increasing LEP energy and reaching at least the energy of $\mathrm{W}$ pair production. It is essentially based on the superconducting (SC) cavity technology developed at CERN since 1979.

The basic choices for the LEP SC cavities were made early in the project: $352 \mathrm{MHz}$ frequency (for compatibility reasons and to minimize the critical transverse impedance of LEP), four-cell structure with couplers on the beam tubes, $4.5 \mathrm{~K}$ operating temperature, modular cryostat with easy access to the cavities and ancillary equipment, thermal and magnetostrictive tuners inside the cryostat. It was also decided that industrial firms would produce the SC cavity modules. The major difference, when compared to other large SC cavity projects (notably CEBAF) is that the cavities would be specified and accepted by CERN according to their RF performance (RF quality factor at the design accelerating field), as measured by CERN.

After an intense period of development it was decided that the LEP2 programme would be based on the niobium copper $(\mathrm{Nb} / \mathrm{Cu})$ technology. The inherent advantages of $\mathrm{Nb} / \mathrm{Cu}$ cavities - much better thermal stability against quenching, savings on $\mathrm{Nb}$ material, insensitivity to small magnetic fields, higher quality factor - turned out to be very welcome in the course of the project.

\section{SC CAVITY PERFORMANCE}
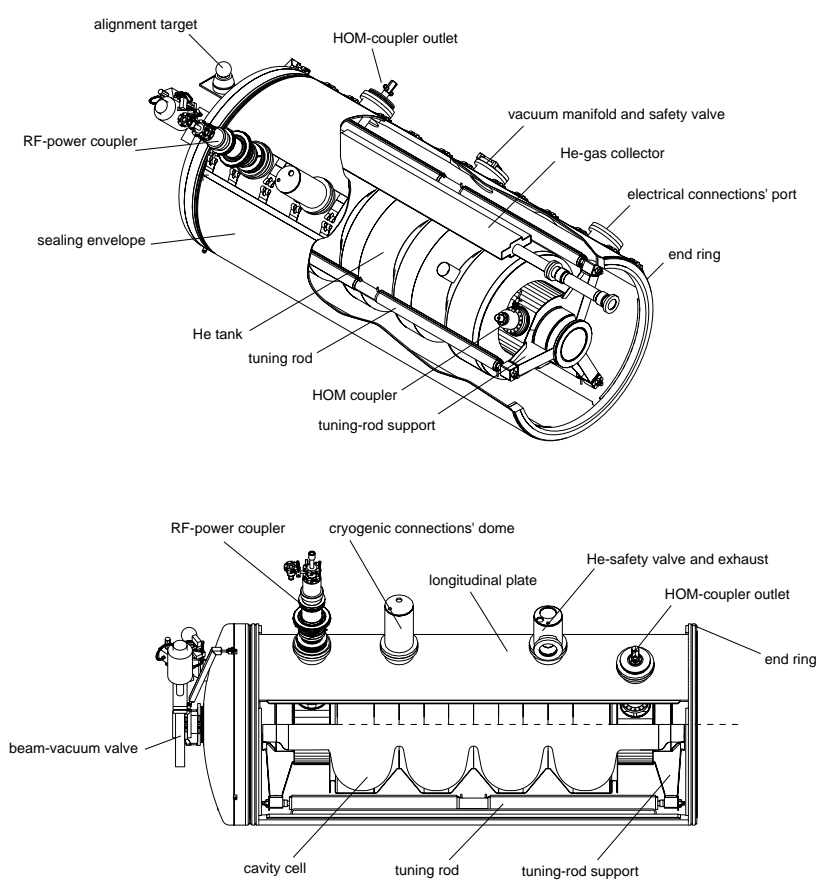

Fig. 1 The LEP2 SC cavity

Fig. 1 shows the four cell LEP2 cavity in its cryostat. The cavity itself surrounded by its helium tank and its three tuner bars is suspended inside the cryostat. The length of the tuner bars is controlled by their thermal expansion and by the magnetostrictive effect. The cryostat, with its three wide-barrel staves provides easy access to the cavity as neither a magnetic shield nor an intermediate thermal shield is necessary. Thermal insulation is achieved with superinsulation mattresses only. Four cavities are assembled together in a common cryostat to form a cryomodule $12.5 \mathrm{~m}$ long, including the end elements [1] [2].

Table 1 shows the major parameters of the LEP2 cavities, the most critical being the quality factor $\mathrm{Q}_{0}$ at the operating field $(6 \mathrm{MV} / \mathrm{m})$. This is the parameter which is measured during the acceptance tests made at CERN of all cavities and modules produced by industry. Fig. 2 shows typical $Q(E)$ curves of cavities fabricated by three different firms following the technique developed at CERN by which a copper cavity is coated inside with a thin niobium film (average thickness $1.5 \mu \mathrm{m}$ ) using a magnetron discharge. This measurement is no longer possible when the RF couplers are installed; however the 
cryogenic losses of a cryomodule can be estimated directly from the cryoplant parameters. The results are coherent with previous RF measurements showing that there is no significant degradation of the cavity performance after mounting of RF couplers, transport and installation in the tunnel.

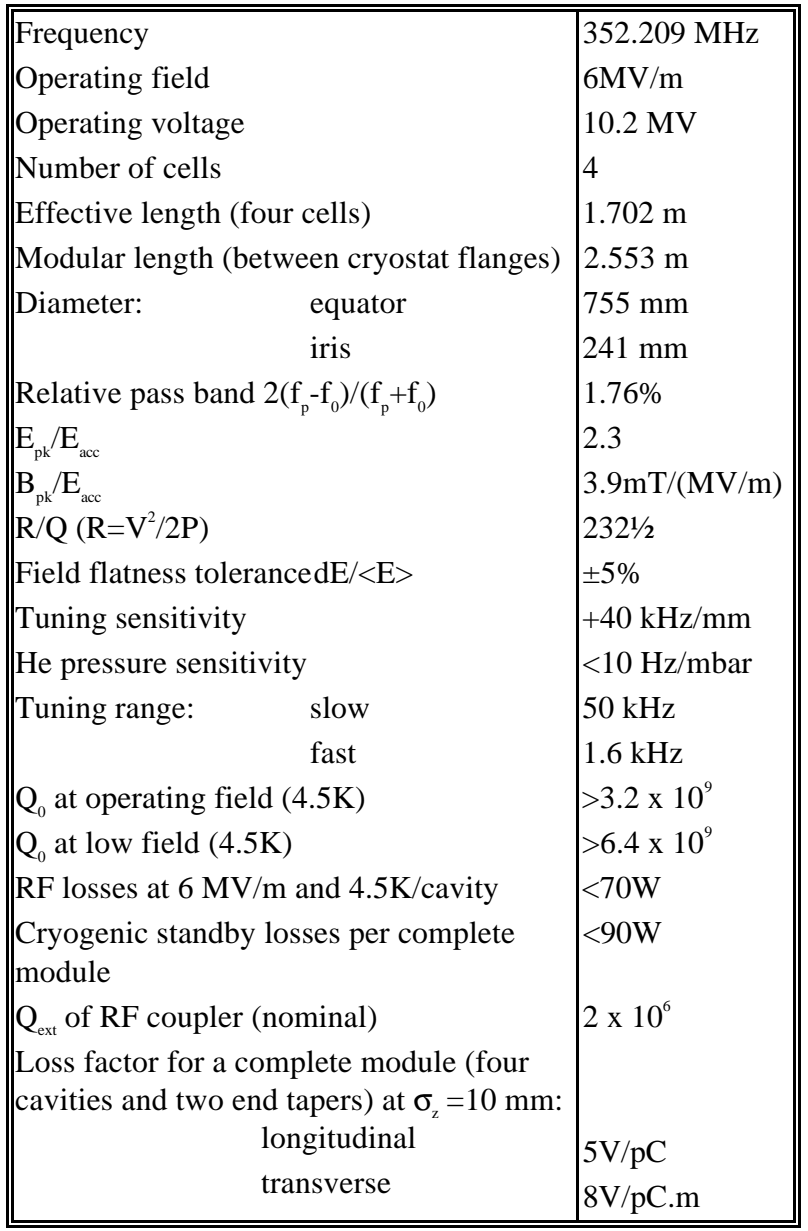

Table 1 LEP2 cavity parameters

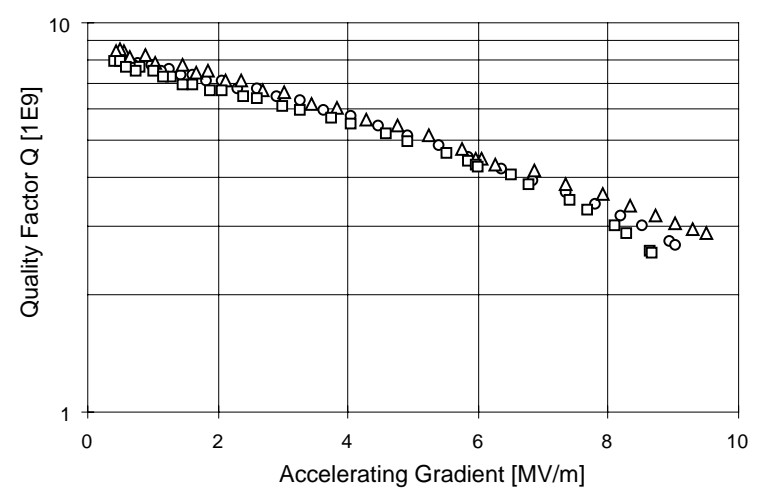

Fig. 2 Best Q vs E curves from three manufacturers

To reach the specified performance helium processing is often used during acceptance tests (without $\mathrm{RF}$ couplers). It has also been applied successfully on a defective cavity in the tunnel, equipped with RF couplers. However, possible discharges, or even arcing in the coupler, are potential risks of this technique. Pulsed power processing (pulses a few ms long, peak fields up to about $8 \mathrm{MV} / \mathrm{m}$ ) is used more commonly both in the power test bench and in the tunnel to reach maximum cavity performance.

A vacuum accident occurred during the last LEP shutdown, where a complete warm module was rapidly vented with filtered nitrogen. Due to a very tight installation and testing schedule it was not possible to recheck the cavities until now. We are eagerly waiting for the new measurements of cavity performance to see whether this module can be recovered in situ or must be taken out of the tunnel for repair. Some years ago, a similar accident happened in the SPS: a cold cavity was slowly vented with unfiltered air; nevertheless, the cavity could still be operated afterwards.

Fig. 3 shows typical radiation levels measured at the end of several modules $15 \mathrm{~cm}$ off axis. The sharp increase with accelerating field indicates a strong field emission. The measured spectrum of the field-emitted electrons extends up to the maximum accelerating voltage of the module $(40 \mathrm{MeV})$ and in some cases leads to activation of elements.

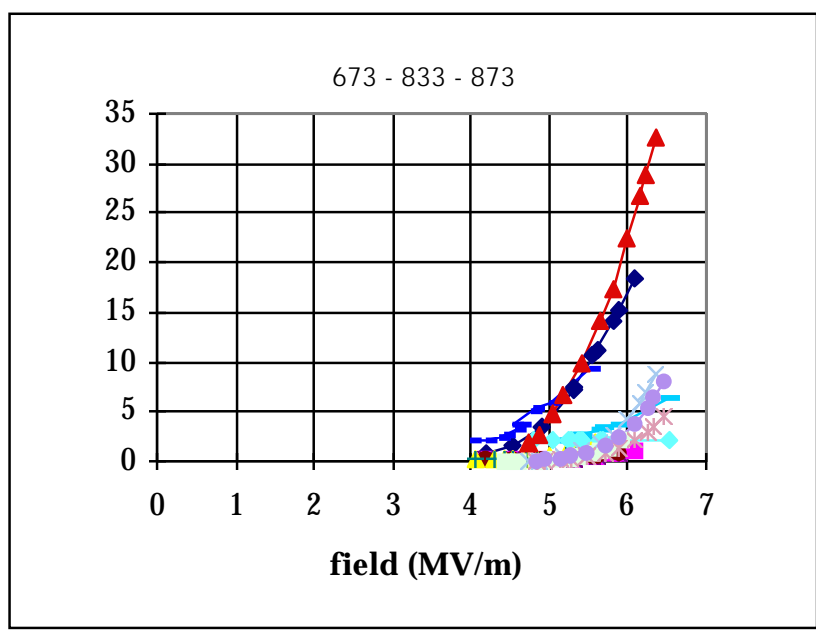

Fig. 3 Typical radiation levels of modules

In the early phases of the project, the main cavity coupler was a very serious cause of concern. Recurrent multipacting discharges plagued the operation of the couplers when installed on the cavities. This is attributed to the strongly enhanced secondary electron emission coefficient of a cold surface if it becomes covered with adsorbed gases. Such a situation occurs on the cold part of the outer conductor of the coaxial coupler (Fig. 4). The problem is cured by baking the window (the source of gas molecules) in situ before cooling the outer conductor of the coaxial line (cooled last) and by applying DC bias $(+2.5 \mathrm{kV})$ on the inner conductor, which completely prevents multipacting during operation [3].

LEP couplers, including their variable version have been extensively tested on a dedicated cold cavity, simulating the real operating conditions. An RF power of $300 \mathrm{~kW}$ has been transferred through the cavity during 
long periods, limited only by the load on the output coupler. Short pulses of $500 \mathrm{~kW}, 1.5 \mathrm{~ms}$ duration have also been transmitted through the test cavity, in pure travelling-wave mode.

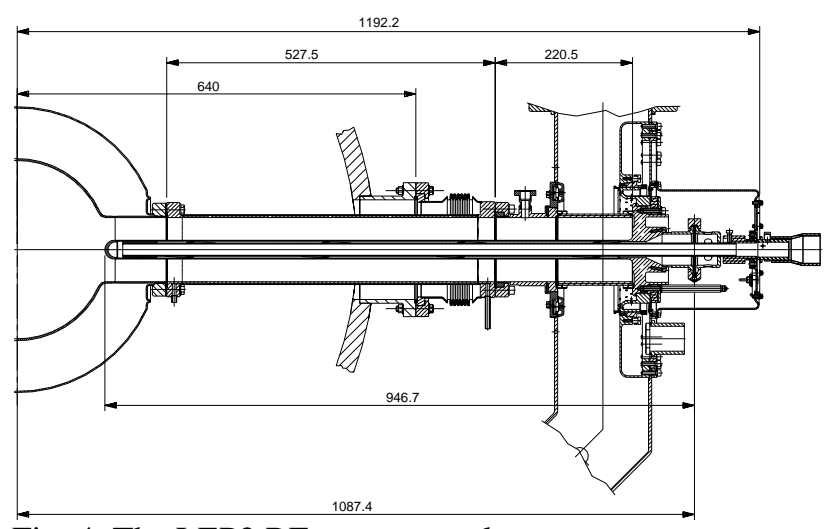

Fig. 4 The LEP2 RF power coupler

The production of cavities and modules by industry is by now almost complete. Table II shows the past and future steps in the installation programme [4].

\begin{tabular}{||l|l|l|l|l||}
\hline & $\begin{array}{l}\text { June } \\
96\end{array}$ & Oct. 96 & $\begin{array}{l}\text { May } \\
97\end{array}$ & $\begin{array}{l}\text { May } \\
98\end{array}$ \\
\hline \# Cu cav & 120 & 120 & 84 & 48 \\
\hline $\mathrm{MV} \mathrm{Cu}$ & 300 & 300 & 215 & 130 \\
\hline \# Nb cav & 4 & 12 & 16 & 16 \\
\hline $\mathrm{MV} \mathrm{Nb}$ & 34 & 102 & 136 & 136 \\
\hline \# Nb/Cu cav & 140 & 160 & 224 & 256 \\
\hline $\mathrm{MV} \mathrm{Nb/Cu}$ & 1434 & 1638 & 2294 & 2621 \\
\hline$\Sigma \mathrm{MV} \mathrm{max}$ & 1768 & 2040 & 2645 & 2887 \\
\hline$\Sigma \mathrm{MV}_{\text {op }}$ & 1600 & 1873 & 2478 & 2720 \\
\hline $\mathrm{E}(\mathrm{GeV})$ & 80.5 & 86 & 92 & 96 \\
\hline
\end{tabular}

Table 2 Number of cavities in LEP and available voltage

\section{MICROPHONICS}

Superconducting cavities, with their narrow bandwidth $( \pm 90 \mathrm{~Hz}$ for LEP2 cavities) are usually prone to microphonic effects. Indeed RF field fluctuations are normally observed on LEP2 cavities at frequencies corresponding to the first mechanical resonances (longitudinal) of the cavity, around $100 \mathrm{~Hz}$. Transverse resonances, occurring at lower frequencies do not seem to be harmful.

A source of mechanical vibration external to the cavity itself was identified and subsequently cured on most modules. It was due to thermoacoustic oscillations occurring in the outlet gas helium connection to the transfer lines, which showed a reduced cross-section to the warming helium gas flow. The solution was to damp this oscillation with a thin kapton ${ }^{\circledR}$ film wrapped around the inner tube.

The other observed effect is intrinsic to the cavity itself and turned out to be more difficult to eliminate. It is a ponderomotive closed loop instability occurring when the cavity resonant frequency is shifted by the tuning system, to keep the load to the RF generator real, in the presence of beam loading [5]. In such a situation, any resonant frequency disturbance results in a disturbance on the field, which itself perturbs the cavity frequency due to radiation "pressure" on the walls. This results in a potentially unstable closed loop. The observed instabilities occur at the first longitudinal resonances of the cavity $(\sim 100 \mathrm{~Hz})$ unfortunately very close to the $-3 \mathrm{~dB}$ points $( \pm 90 \mathrm{~Hz})$ of the cavity bandwidth. They are strongly dependent on the cavity fields and on beam current (via the cavity detuning), and at present are a major limitation for LEP2.

A study has been launched to actively damp the mechanical resonances of the cavity, acting on the fast magnetostrictive tuner. The difficulty here is to damp simultaneously two very close resonances (separated by about 5 to $10 \mathrm{~Hz}$ ) with a robust enough system. The problem, in principle disappears if one keeps the cavity exactly on tune with the RF frequency, irrespective of beam loading. The additional RF power consumption in this case is perfectly acceptable $(4.25 \mathrm{~kW}$ per cavity at $90 \mathrm{GeV}$ and $10 \mathrm{~mA}$ for the LEP2 parameters). However the RF field in the coupler increases significantly, but as explained before, a large safety margin is available in the couplers and this should not be a problem.

Actually, by injecting a phase offset in the tuner phase detector, one can suppress the instability for a given voltage and current. However the cavity phasing errors, the dispersion in coupling factors and cavity fields makes it rather dificult to program the tuner offsets especially during ramping energy and field. In particular, a common offset cannot be simply applied to all eight cavities driven by the same klystron. Despite its limitations and difficulties, this is the technique presently used to avoid ponderomotive instabilities as much as possible.

Another method is being actively tested where a cavity is excited mechanically, via the fast tuner, at a single frequency (at present $40 \mathrm{~Hz}$ ). The $40 \mathrm{~Hz}$ cavity voltage modulation detected by a synchronous demodulator provides an error signal when the cavity is out of tune. This signal can replace the usual phase detector signal (phase between cavity field and klystron drive) to close the tuning servo loop. Such a system (already tested without beam) is independent of beam current and insensitive to phasing errors; it keeps the cavity always on tune. The cavities would be modulated by pairs, with opposite phases, to cancel out the residual phase modulation at $40 \mathrm{~Hz}$ (further reduced by the "vector sum feedback").

\section{HIGHER ORDER MODES}

Each LEP2 cavity is equipped with two higher order mode (HOM) couplers. They are of the "hook" type where a series notch filter at the RF frequency is established with the inductance of the "hook" and its capacitance to the wall of the cavity port [6]. The connecting RF line between the cold coupler and the cryostat wall is a $25 \Omega$ "rigid" coaxial line made of two thin stainless steel, copper plated tubes. Finger contacts 
at either end of the line allow some mechanical displacements during cool down.

It has been demonstrated experimentally that more than $850 \mathrm{~W}$ can be transmitted through the HOM coupler and its line at $630 \mathrm{MHz}$ (frequency of the dominant longitudinal HOM of the LEP2 cavity). This figure is beyond what is expected in LEP2 operation. However, it has been observed that many HOM loads failed during operation, due to transients. They are now being replaced by long, lossy RF cables.

Initial measurements of power deposited by single bunches in the HOM coupler loads have confirmed the expected value of the loss factor of the cavities $\left(0.44 \mathrm{~V} / \mathrm{pC}\right.$ at $\sigma_{\mathrm{z}}=16 \mathrm{~mm}$, excluding the fundamental).

Above $2.2 \mathrm{GHz}$ (cut-off frequency of the $10 \mathrm{~cm}$ diameter beam tube) HOM power may propagate outside the SC cavity module. Using calorimetric measurements on RF ferrite absorbers installed in the LEP vacuum chamber (one close to an SC module, one far away), the first experimental evidence of HOM power radiated outside the SC modules was obtained.

During the last run of 1996, it was observed that the cryogenic consumption of the modules was increasing with higher beam current and shorter bunches, leading to the suspicion of HOM trapped inside the 4-cavity structure. A concomitant increase of the temperature of the cold intercavity bellows (copper plated stainless steel, diameter $20 \mathrm{~cm}$ ) has also been observed and supports this hypothesis. An energy dependence of the power deposited in standard warm bellows outside the modules has also been noted, without any convincing explanation for the moment. Synchrotron radiation, as well as the radiation coming out of the modules as a result of field emission, has been considered, but obviously more measurements are needed to analyse these effects.

\section{RF POWER SYSTEM}

The LEP RF system is based on high power klystrons (1.3 MW), each feeding $8 \mathrm{SC}$ cavities via a circulator and a series of symmetrical magic tee splitting stages [7]. The circulator is terminated on its third port by a $300 \mathrm{~kW}$ water load; each magic tee is terminated by a $100 \mathrm{~kW}$ load. To improve crosstalk between adjacent cavities, better matching of the $100 \mathrm{~kW}$ loads is required. The solution which is being implemented now is to dope the cooling water of the load with sodium nitrite $\left(\mathrm{NaNO}_{2}\right)$.

The 24 klystrons of the SC units have so far accumulated between 1000 and 9000 operating hours, with an average of 3500 hours. The average lifetime of these high power klystrons is expected to be 18000 hours (DESY statistics); in the LEP copper RF system nine tubes reached more than 28000 hours. We therefore do not expect klystron problems in the near future.

The reflected wave peak power from a SC cavity when the RF source is abruptly switched off amounts to four times the zero beam current forward power (1 MW in the LEP2 case). It was found that these high power bursts (duration in the ms range) can induce arcs in the line and RF transformer connecting the $300 \mathrm{~kW}$ load to the circulator. The arcs eventually propagated to the circulator itself; two of them had to be changed. The solution is to replace the coaxial elbows and wave guide transformer between circulator and $300 \mathrm{~kW}$ load by waveguide components and to improve the klystron reflected power interlock. In addition, fast detuning of unused cavities is implemented to avoid strong beam induced power reaching the circulator.

\section{CONTROLS AND OPERATION}

In addition to the classical regulation circuits, notably the slow amplitude loop acting via the DC current and hence the RF gain, the LEP2 klystrons are now equipped with an RF "vector sum" feedback loop (Fig. 5). The total RF voltage seen by the beam when crossing the eight cavities driven by a common klystron is reconstructed from the fieldprobe signals of each cavity. Great care must be applied to the calibration of the probes and cable connections (in amplitude and phase) to ensure that the overall vector sum signal is a faithful representation of the RF voltage experienced by the beam. The "vector sum" signal is maintained equal to the demanded RF voltage by the action of the RF feedback loop.

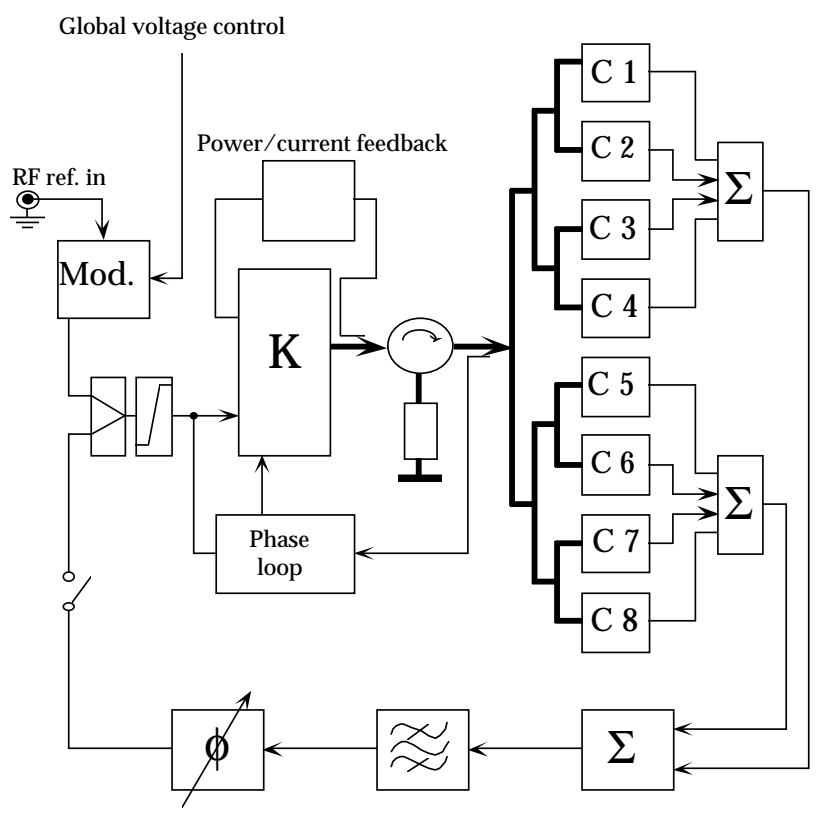

Fig. 5 Simplified block diagram of the vector-sum feedback

The open loop gain of the vector sum feedback RF loop has been adjusted to $26 \mathrm{~dB}$ for a klystron current of 10 A but it varies linearly with the klystron current. It is actually limited by the precision with which the vector sum can be constructed.

The loop gain is sufficient to reduce the equivalent impedance of the SC cavities so that cavity coupling via the beam becomes negligible and the operating conditions of the RF system remain far from the Robinson threshold. The phase deviations of the cavities (either from residual microphonics or from various phase settings of the servo tuners) are corrected by the vector sum RF feedback, as well as the cavity overvoltage when the beam is suddenly lost. 
The minimum attainable RF voltage, at the injection energy of $22 \mathrm{GeV}$ is limited by the tuning loops of the cavities. These loops are sensitive to spurious signals from RF ripple, crosstalk between cavities, finite directivity of the directional couplers and to imperfect phasing of the cavities. The associated problems have been largely alleviated by running at a higher voltage (450 MV, $\left.\mathrm{Q}_{\mathrm{s}}=0.14\right)$ at injection, with all cavities turned on.

With the experience gained in running the RF system in 1996 it is now possible to envisage automation of certain procedures. Application software to perform automated checks of cavity tuning and unit phase could be provided, as could an automated procedure for optimising the tuner setpoints to suppress ponderomotive oscillations. The major candidate for automation from the operational point of view is, however, the automatic switch-on of tripped units.

In 1996 it was standard procedure to stop the ramp a few $\mathrm{GeV}$ below physics energy. The RF voltage was then ramped to its operational maximum, any tripped RF units restarted and any ponderomotive oscillations controlled by means of tuner adjustments. Only when the RF system was stable was the machine ramped to physics energy.

There is a significant spread in the voltages produced by the eight cavities driven by a common klystron. This indeed limits the maximum voltage available and is due to differences in cavity coupling factors $\left(\mathrm{Q}_{\mathrm{ext}}\right)$ and possible asymmetries in the waveguide and magic tees distribution. A very simple $\lambda / 4$ transformer can be inserted in the waveguide to compensate for large variations of $\mathrm{Q}_{\mathrm{ext}}$ (this has been successfully tested, even with beam). With high beam loading (especially at injection) additional cavity voltage dispersion results from differences in waveguide electrical length or longitudinal position of the cavities inside the cryostat. To correct all these effects will require tedious adjustments, which were not really possible during the last busy years.

\section{CONCLUSION}

The largest superconducting RF system in the world is now in operation. The energy of LEP has been pushed from $45 \mathrm{GeV}$ up to $86 \mathrm{GeV}$ and physics above the $\mathrm{W}$ pair production threshold has started. The SC cavities and couplers behaved in the machine as expected; no degradation of performance was recorded. Final adjustments of the RF system will be a tedious, but straightforward task, and it seems that the control of ponderomotive instabilities is within reach. Finally the overall performance of the RF system might be limited by the capacity of the cryoplants. Upgrading of the installed cooling capacity, which was anyhow planned for LHC, might therefore be implemented earlier than foreseen (hopefully in 1999) to the benefit of LEP2.

\section{ACKNOWLEDGEMENTS}

Finally, it is a pleasure to associate to this presentation all the team who engineered, constructed and put into operation the LEP2 RF system and whose competence, dedication and long-lasting enthusiasm were essential to its success.

\section{REFERENCES}

[1] 'The LEP design report', vol. III, LEP2, C. Wyss, editor, CERN AC/96-01, LEP2

[2] D. Boussard: 'Operational experience with the LEP2, S.C. cavity system', Proc. 5th EPAC, Sitges (Barcelona, Spain), p. 187

[3] H. P. Kindermann: 'Status of RF power coupler for superconducting cavities at CERN', ibid. p. 2091

[4] E. Chiaveri: 'Large scale industrial production of superconducting cavities', ibid. p. 200

[5] D. Boussard, P. Brown, J. Tückmantel: 'Electroacoustic oscillations in the LEP S.C. cavities', ibid. p. 2097

[6] E. Haebel: 'Couplers for cavities'. Proc. CAS, Hamburg 1995, S. Turner, editor, CERN 96-03 (1996), p. 231

[7] H. Frischholz: 'The LEP2 RF power generation system'. Proc. IEEE PAC, Washington 1993, p. 1247 and CERN SL/93-20 